J.-M. Tschopp ${ }^{1}$

W. Frank ${ }^{2}$

M. Noppen ${ }^{3}$

\section{Das Management des Spontanpneumothorax}

The Management of Spontaneous Pneumothorax

\section{Zusammenfassung}

Das optimale Management des Spontanpneumothorax (SPTX) beinhaltet konservative wie interventionelle, internistische wie chirurgische Optionen, bleibt aber hinsichtlich Akutbehandlung und Präventivstrategien wegen unzulänglicher Datenlage z.T. umstritten. Nach aktuellen Konsensusempfehlungen kann beim gering ausgedehnten und asymptomatischen Pneumothorax die Spontanresorption abgewartet werden. Ausgedehnte und symptomatische Pneumothoraxes können zu etwa $2 / 3$ mit einer einfachen Aspiration oder englumigen Kathetern $(<16 \mathrm{~F})$ erfolgreich behandelt werden. Die höchste Erfolgsquote bieten mit bis zu $96 \%$ weitlumige Drainagen ( $>20 \mathrm{~F}$ ), die beim Versagen der einfachen Absaugung und bei Patienten mit sekundärem SPTX insbesondere im fortgeschrittenen Lebensalter bereits primär zu empfehlen sind - am besten in Verbindung mit der Thorakoskopie (Pleuroskopie). Eine Präventivtherapie ist spätestens beim 1. Rezidiv und primär beim Nachweis prädisponierender pleuropulmonaler Veränderungen wie beim sekundären PTX indiziert. Die derzeitige Datenlage erlaubt bezogen auf die Effektivität als primärer Endpunkt keine klare Präferenz zwischen einer internistischen Talkpoudrage und chirurgischen Strategien (üblicherweise VATS-gestützte partielle Pleurektomie \pm Bullaablation bzw. Parenchymresektion). Die Therapieentscheidung bleibt daher ermessensabhängig. Die internistische thorakoskopische Talkpleurodese ist jedoch als hocheffektiv und sicher in der Prävention von Rezidiven anzusehen. Hierfür sprechen auch die niedrigen Kosten, die praktisch denen einer konservativen Behandlung mittels weitlumiger Pleuradrainage entsprechen.

\section{Abstract}

The optimum management of spontaneous pneumothorax (SPTX) includes medical and surgical approaches but remains somewhat controversial due to inconclusive data background both in the treatment of the acute episode and preventive strategies. Current consensus recommendations suggest, that small and asymptomatic pneumothoraces can be managed conservatively by observation. Large and symptomatic pneumothoraces will respond to simple aspiration or small size catheter $(<16 \mathrm{~F})$ insertion in about $2 / 3$ of cases. Otherwise, and in secondary SPTX, particularly in the elderly patient, large size ( $>20 \mathrm{~F}$ ) chest drainage, optimally combined with thoracoscopy (pleuroscopy), is the treatment of choice allowing success rates up to $96 \%$. Preventive strategies are indicated after the first recurrence and when predisposing pleuropulmonary lesions as in secondary SPTX are evident. With efficacy as a primary endpoint, currently available data do not provide clear evidence-based preference criteria between medical thoracoscopic talc pleurodesis and surgical strategies (usually VATS-based partial pleurectomy \pm parenchymal resection). Therefore selection of the appropriate procedure remains influenced by expert opinion. However simple talc poudrage under medical thoracoscopy has been shown to be highly effective, safe and cheap at a cost level virtually not exceeding that of large size chest drainage. 
Der Spontanpneumothorax (SPTX) ist kein sehr häufiges, oft aber ein kritisches Ereignis. Fragen des Managements schließen sowohl abwartend-konservative als auch aktive, unterschiedlich invasive Konzepte ein und adressieren so gleichermaßen Internisten und Chirurgen, Allgemeinmediziner und Spezialisten wie etwa Notfall- und Intensivmediziner. Vor dem Hintergrund oft differierender Leitlinien für das rationale Management des Akutszenarios und der Rezidivprävention erscheint es gerechtfertigt, die Behandlungsempfehlungen des SPTX anhand aktueller evidenzbasierter Daten kritisch zu überprüfen und ggf. zu modifizieren. Hierbei soll sich die Darstellung auf den primären und sekundären Spontanpneumothorax (PSPTX bzw. SSPTX) konzentrieren. Der traumatische Pneumothorax wird nur soweit einbezogen, als er iatrogen, unter anderem auch unter maschineller Beatmung auftritt. Es hat zahlreiche Versuche und Vorschläge gegeben, zu einem standardisierten Vorgehen beim SPTX zu kommen [1 -3]. Dessen ungeachtet bleibt die optimale Behandlung wegen der geringen Zahl kontrollierter prospektiver Studien, aber auch wegen noch bestehender pathogenetischer Unklarheiten, ein kontroverses Thema. Dabei gilt die Prämisse, dass in Anbetracht der klinischen und ätiologischen Heterogenität des Krankheitsbilds entsprechend abgestufte internistische, interventionelle und chirurgische Vorgehensweisen erst durch enge interdisziplinäre Zusammenarbeit in einem entsprechend strukturierten Zentrum optimal realisiert werden können.

\section{Pimärer Spontanpneumothorax (Psptx)}

\section{Klinische Inzidenz und Manifestationen}

Der primäre Spontanpneumothorax (PSPTX) betrifft vor allem klinisch gesunde junge Menschen mit einem Altersgipfel zwischen 20-30 Jahren und weist eine Jahresinzidenz von 7,4-18 Fällen pro 100000 Personen in der männlichen Bevölkerung und von 1,2-6 Fälle pro 100000 in der weiblichen Bevölkerung auf [4-6]. Die höchste Inzidenz des PSP findet sich bei jungen hochgewachsenen und leptosomen männlichen Personen, was in der französischen Literatur den Ausdruck „pneumothorax du jeune conscrit“ („Wehrpflichtigen-Pneumothorax“) geprägt hat. Der Pneumothorax äußert sich meist durch einseitige, stechende thorakale Schmerzen, oft steht Dyspnoe im Vordergrund, seltener Husten. Entgegen einer verbreiteten Meinung spielt körperliche Anstrengung nahezu keine auslösende Rolle, vielmehr bestehen Zusammenhänge mit atmosphärischen Druckschwankungen [7]. Auf denselben Mechanismus sind mit Wahrscheinlichkeit auch selten beschriebene primäre Pneumothoraxepisoden im Zusammenhang mit niedrigfrequenter lauter Musikeinwirkung zurückzuführen [8]. Tabakgenuss ist der entscheidende exogene Faktor: das Risiko im Verlaufe seines Leben einen Pneumothorax zu entwickeln, steigt beim Raucher ungefähr auf $12 \%$, beim Nichtraucher liegt es vergleichsweise bei nur 0,1\% [9]. Die meisten Patienten mit PSPTX sind in der Tat Raucher [9].

\section{Diagnose und klinische Bewertung}

Die Diagnose wird klinisch vermutet und im Allgemeinen durch eine anteroposteriore Röntgenaufnahme des Thorax in Inspiration bestätigt (Abb.1). Der Wert einer zusätzlichen Exspirationsaufnahme für die Aufdeckung des SPTX ist nicht gesichert [10].

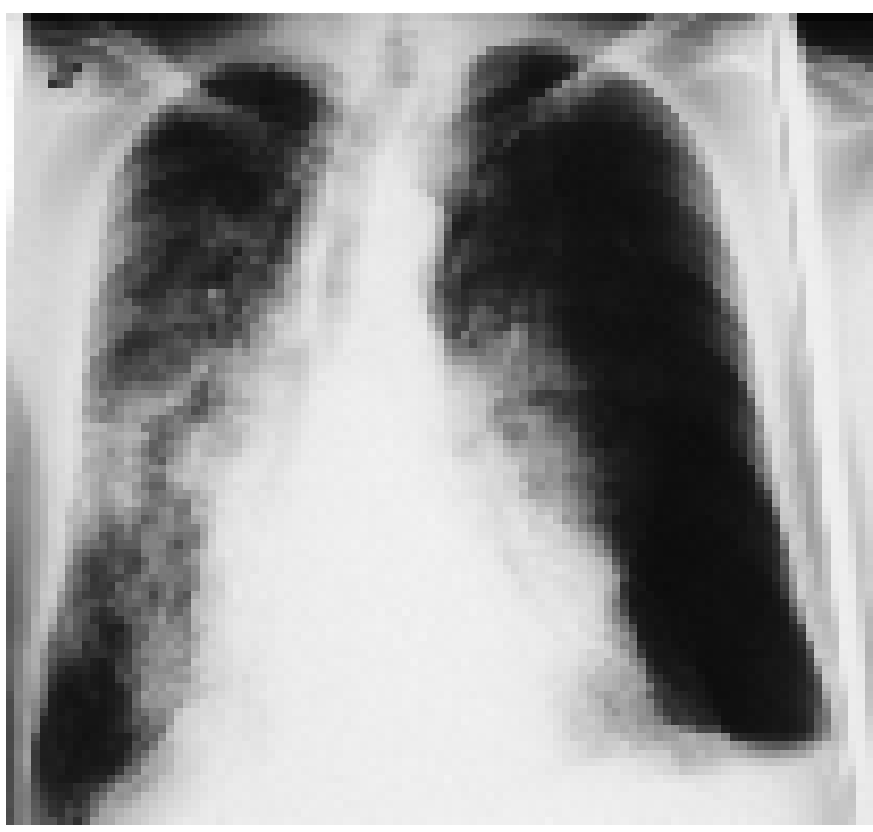

Abb. 1 Spannungspneumothorax bei COPD-Patient. Die Differenzialdiagnose zum großbullösen Lungenemphysem kann bei nativ nicht erkennbarer Lungenkontur, wie im Beispiel, schwierig sein.

Hingegen erbringt die zusätzliche Aufnahme in seitlicher Projektion in $14 \%$ der Fälle diagnoseweisende Informationen [11]. Wichtig ist, bei Risikopatienten mit pulmonalen Vorerkrankungen bzw. maschineller Beatmung immer an die Möglichkeit eines PTX zu denken, und relevante Differenzialdiagnosen (kardiovaskuläre und gastrointestinale Erkrankungen) auszuschließen. Eine Computertomographie kann beim Verdacht auf großbullöses Lungenemphysem differenzialdiagnostisch eingesetzt werden und vermeidet in diesem Falle eine kontraindizierte und gefährliche pleurale Intervention [12].

\section{Pathophysiologie des primären Spontanpneumothorax}

Sattler hat 1937 basierend auf umfangreichen thorakoskopischen Untersuchungen blasige Lungenveränderungen (sog. „blebs“) als prädisponierend für den Spontanpneumothorax identifiziert [13] und die Grundlage zu der traditionellen Anschauung gelegt, dass die Ruptur von „Blasen“ ursächlich für das Pneumothoraxereignis sei. Viel später konnte im Zuge der Fortschritte der bildgebenden Verfahren und der Videothoraxkoskopie gezeigt werden, dass der größte Teil der jungen Patienten mit PSPTX im CT diskrete Schädigungen des Typs „emphysema like changes“ (ELC) aufweist und zwar nicht nur auf der Seite des Pneumothorax, sondern auch in der mutmaßlich gesunden kontralateralen Lunge $[14,15]$. Chirurgen hatten bereits zuvor in der Behandlung des Spontanpneumothorax durch Sternotomie die Beobachtung gemacht, dass auch in der kontralateralen Lunge subpleurale „blebs“ in 79-96\% der Fälle vorhanden sind $[16,17]$. Licht- und elektronenoptische Untersuchungen von Lungenresektaten beim PSPTX zeigten indes nur selten die erwartete Ruptur der viszeralen Pleura, sondern häufig nur mikroskopische pleuropulmonale Kommunikationen im Umfeld der ELCs oder an normal erscheinenden Lungenanteilen $[18,19]$. Thorakoskopische Longitudinalstudien haben keine Zunahme oder anderweitige Lokalisationen von „blebs“ im Falle des Rezidivs nachweisen können [20]. Bei jungen Nichtrauchern ohne alpha-1-Antitryp- 
sin-Mangelsyndrom und einer Vorgeschichte von PSPTX liegt die ELC-Inzidenz bei $80 \%$, bei Personen ohne Pneumothorax-Vorgeschichte in der "matched pair"-Analyse hingegen nur bei $20 \%$ [21]. Die ELCs stellen somit einen pathologischen Prozess dar, der die Lunge und Pleura in diffuser Weise involviert und sicher in der Pathogenese beteiligt ist. Nach Schramel u. Mitarb. [22] ist davon auszugehen, dass der Spontanpneumothorax auf dem Boden einer Entzündung der kleinen Atemwege entsteht. Die Ruptur und Luftdissektion dürfte in den kleinen Atemwegen im Umfeld der ELCs erfolgen [23]. Von dort kommt es zum Lufteintritt in das subpleurale Interstitium mit konsekutivem Pneumothorax, ferner in das bronchovaskuläre Interstitium mit potenzieller Verteilung bis ins Mediastinum (interstitielles und Mediastinalemphysem) [24]. Nach allen vorliegenden Untersuchungen und dem gegenwärtigen Wissen über die Pathophysiologie des primären Spontanpneumothorax muss man entgegen einer verbreiteten Anschauung davon ausgehen, dass das Luftleck nicht notwendigerweise mit der Lokalisation größerer Blasen und sichtbarer „blebs“ identisch ist, sondern sich irgendwo auf der Pleura befindet $[25,26]$. Daraus wäre im Vorgriff auf die Therapie abzuleiten, dass die systematische Resektion der sichtbaren Luftblasen nicht unbedingt gerechtfertigt ist, beziehungsweise dass sie in der Behandlung des Pneumothorax über die einfache Pleurodese hinaus keinen zusätzlichen Nutzen erbringt [27-30].

\section{Sekundärer Spontanpneumothorax (SSPTX)}

\section{Klinische Inzidenz und Manifestationen}

Im Gegensatz zum primären Spontanpneumothorax verläuft der SSPTX, der mit einer latenten oder manifesten Lungenkrankheit assoziiert ist, durch die Dekompensation der pulmonalen Grundkrankheit oft lebensbedrohlich [24,31,32]. In den 1980er-Jahren hat die AIDS-Pneumonitis durch Pneumocystis carinii erheblich zur SSPTX-Inzidenz beigetragen [33]. Gegenwärtig ist die Hauptursache die chronisch obstruktive Lungenkrankheit (COPD), gefolgt von interstitiellen Lungenkrankheiten (ILD) und der zystischen Fibrose (CF) mit einem Risiko, das jeweils proportional zur Schwere der funktionellen Einschränkung zunimmt [34]. Nahezu $1 / 3$ der Fälle weist eine massive Einschränkung des $\mathrm{FEV}_{1}$ $(<40 \% \mathrm{FVC})$ auf [35].

Die Inzidenz des sekundären Spontanpneumothorax beträgt ungefähr 6 Fälle auf 100000 Einwohner pro Jahr [4,5]. Die höchste Inzidenz ist im Alter von 60 Jahren zu verzeichnen und geht damit parallel zur Prävalenz der COPD in der gesamten Bevölkerung.

\section{Klinische Präsentation}

Im Gegensatz zum PSPTX ist eine Dyspnoe bis hin zu Orthopnoe fast immer vorhanden. Die Komplikationsrate und -möglichkeiten sind beim sekundären Spontanpneumothorax ungleich gravierender als beim primären (Tab.1). Bedrohliche Komplikationen wie der Spannungspneumothorax und das Mediastinalemphysem gehen nahezu ausnahmslos zulasten des sekundären Spontanpneumothorax bzw. auch des respiratorassoziierten Pneumothorax (RAPTX). Bei schwer eingeschränkter Lungenfunktion oder beim beatmeten Patienten kann selbst ein kleiner Pneumothorax für den Kranken zu einem lebensbedrohlichen Risiko werden. Dies wird durch blutgasanalytische Daten bestätigt, die oft eine schwere Hypoxämie zeigen $\left(\mathrm{PaO}_{2}<6,65 \mathrm{kPa}\right.$ bzw.
Tab. 1 Komplikationen des Spontanpneumothorax

\begin{tabular}{|lll}
\hline Komplikation & $\begin{array}{l}\text { Häufigkeit \% } \\
\text { PSP }\end{array}$ & $\begin{array}{l}\text { Häufigkeit \% } \\
\text { SSP }\end{array}$ \\
\hline Spannungspneu & $<1$ & ca. 20 \\
\hline bronchopleurale Fistel & 4 & ca. 20 \\
\hline bilateraler Pneu & $<2$ & bis $40^{*}$ \\
\hline Kammerung & 4 & k. A. \\
\hline Mediastinal- und Weichteilemphysem & $1-2$ & ca. 20 \\
\hline Reexpansionsödem & 0,5 & k. A. \\
\hline Pyopneumothorax & k. A. & k.A. \\
\hline Hämothorax & $1-2$ & bis 8 \\
\hline Atelektase/Pneumonie & 4 & $>30$ \\
\hline gesamt & ca. 10 & $40-60$ \\
\hline
\end{tabular}

$50 \mathrm{mmHg}$ [ [31]. Die klinische Untersuchung kann täuschen: im Falle einer COPD-Exazerbation, vor allem bei thorakalen Schmerzen muss immer an die Möglichkeit eines Pneumothorax gedacht werden [24,32]. Die Rückfallquote nach dem SSPTX-Erstereignis ist ungleich höher als beim PSPTX. Sie liegt in der Größenordnung von $39-47 \%$ bei COPD und von $50-83 \%$ bei der zystischen Fibrose, erreicht aber nach einem Rezidiv bereits Werte um 90\% [35-37].

\section{Die Behandllung des Spontanpneumothorax}

Für das Management des Pneumothorax gibt es zahlreiche unterschiedlich invasive Optionen entsprechend Tab. 2 wie sie auch in kombinierter Anwendung - in Schlüsselarbeiten diskutiert werden $[22,30,38,39]$. Die Therapie verfolgt dabei zwei wesentliche Ziele: die Entfernung der Luft aus der Pleurahöhle und die Verhinderung des Rezidivs.

\section{Akutbehandlung}

Die Optionen der Akutbehandlung bestehen in der reinen ambulanten Beobachtung, ggf. symptomatischer Therapie (Analgetika) und in der Evakuierung des Pleuraraums. Der gelegentlich gegebenen Empfehlung von low-flow- $\mathrm{O}_{2}$-Gaben liegt die erwar-

Tab. 2 Therapeutische Optionen beim primären Spontanpneumothorax

\begin{tabular}{|c|c|c|}
\hline \multirow[t]{2}{*}{ konservativ } & \multicolumn{2}{|c|}{ interventionell } \\
\hline & internistisch & $\begin{array}{l}\text { chirurgisch } \\
\text { (VATS, Minithorakotomie, } \\
\text { formale Thorakotomie) }\end{array}$ \\
\hline $\begin{array}{l}\text { >Beobachtung } \\
\text { ambulant/klinisch } \\
\pm \text { Analgetika } \\
\pm \text { low-flow- } \mathrm{O}_{2}\end{array}$ & 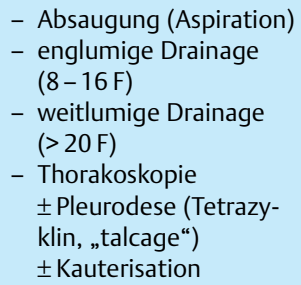 & $\begin{array}{l}\text { - Resektion von Blasen } \\
\text { und Bullae, } \\
\text { - Kauterisation } \\
\text { - Strang- u. Adhäsionslö- } \\
\text { sung } \\
\text { - Pleurodese } \\
\text { - Pleurektomie }\end{array}$ \\
\hline
\end{tabular}


tete Beschleunigung der physiologischerweise niedrigen Luftbzw. $\mathrm{N}_{2}$-Resorptionsrate von $1,2-1,8 \%$ pro Tag zugrunde, was bei einem Pneumothorax einer Ausdehnung bis zu 15\% immerhin eine spontane Resorptionsdauer von 10-14 Tagen bedeuten würde [40]. Low-flow- $\mathrm{O}_{2}$-Gaben stellen somit einen rationalen Ansatz dar, klinisch validiert ist ihr Wert aber nicht. Die Indikation zur Evakuierung mittels einfacher Aspiration oder Drainage wird beim symptomatischen PTX und bei einer Ausdehnung von $>15 \%$ des Hemithoraxvolumens gestellt $[1,40]$. Dies entspricht im Allgemeinen einem apikalen Pneuspalt von $>2 \mathrm{~cm}$ $[41,42]$. Die Erfolgsquoten unterschiedlicher Evakuierungsmethoden sind in Tab. 3 zusammengefasst. Als Erfolgskriterium dient dabei die Erzielung der vollständigen Lungenausdehnung innerhalb von 7 Tagen und dann anhaltend über mindestens 30 Tage. Es handelt sich in Tab. $\mathbf{3}$ meist um pauschale Angaben aus gepoolten Daten ohne Alters- und Geschlechtsdifferenzierung. Tatsächlich spielt zumindest das Alter für die Wahl des Evakuierungsverfahrens jedoch eine Rolle, insofern die Erfolgsraten der Lungenexpansion für den SSPTX nicht nur generell schlechter sind, sondern auch eine deutliche Altersabhängigkeit besteht. So fiel in mehreren Fallserien die Drainageerfolgsrate in der Altersgruppe $>50$ Jahre mit $19-31 \%$ gegenüber der Altersgruppe $<50$ Jahre mit $70-81 \%$ erheblich ab [43-48].

Die einfache Nadelaspiration kann in Lokalanästhesie im 2.-3. ICR ventral in der Medioklavikularlinie mittels einer Teflonkanüle oder eines kurzstreckigen 14 - 16 F-Katheters erfolgen

Tab. 3 Erfolgsquoten der konservativen bzw. internistischen Therapie. Definition des Erfolgs: PTX-Liquidation innerhalb 7 (max. 10) Tagen und mindestens 30 Tage anhaltend

\begin{tabular}{lll}
\hline Management & primärer SPTX & sekundärer SPTX \\
\hline $\begin{array}{l}\text { Beobachtung, } \mathrm{O}_{2} \\
\text { Aspiration }\end{array}$ & k.A. & k.A. \\
\hline $\begin{array}{l}\text { dünnlumige Katheter } \\
\text { ( } \leq \text { Ch 14) }\end{array}$ & $59-83 \%$ & $31-67 \%$ \\
$\begin{array}{l}\text { Thoraxdrainagen } \\
(\geq \text { Ch 20) }\end{array}$ & $93-94 \%$ & $80 \% *$ \\
\hline
\end{tabular}

Noppen 2002, Baumann 2000, Conces 1998, Miller 1996, Light 1993, Matthys 1988, Videm 1987, Withers 1964

${ }^{*}$ inklusive multipler Drainagen
(Abb. 2). Sobald nach Anschluss an ein Saugsystem und ein Wasserschloss die Lunge expandiert und der Fisteldurchfluss mindestens 30 Minuten zum Erliegen gekommen ist, wird die Kanüle bzw. der Katheter wieder entfernt $[36,47,49]$. Für Verweilkatheter, insbesondere aber weitlumige Drainagen ist eine adäquate Einlagetechnik zweifellos eine wichtige Erfolgsvoraussetzung. Hier ist der angesprochene, in der Notfallmedizin häufig empfohlene und praktizierte apikoventrale Zugang der Abb. 2 keineswegs als optimal anzusehen, da er einen zu kurzstreckigen und transversalen intrathorakalen Verlauf des Katheters bzw. Drains zur Folge hat mit der Gefahr des Aufstauchens und des inneren und äußeren Abknickens [38,41,42]. Bei zu medialem Zugang ist die Verletzung der a. thoracica interna nicht ausgeschlossen. $\mathrm{Zu}$ empfehlen und für weitlumige Drainagen zwingend, ist vielmehr der 4. - 5. -6. ICR in der mittleren bis vorderen Axillarlinie, der endothorakal den funktionell optimalen langstreckig-parietalen Drainageverlauf begünstigt (Abb. 2) $[38,41,42]$.

Das optimale Kaliber von Thoraxdrainagen stellt immer wieder einen Gegenstand der Kontroverse dar. Grundsätzlich sollte zwischen englumigen Drains bzw. Kathetern $(\leq 16 \mathrm{~F})$ und weitlumigen Drainagen $(\geq 20 \mathrm{~F})$ unterschieden werden, wobei gilt: $1 \mathrm{~F}($ French $)=1 \mathrm{Ch}$ (Charriere) $=0,33 \mathrm{~mm}=0,0131$ inch. Der unkomplizierte, zumal primäre SPTX kann, belegt durch gute Daten erfolgreich mit 8-14 F-Kathetern behandelt werden $[38,39,41-50]$. Diese ermöglichen je nach Durchmesser, Design und Fenestrierung einen ausreichenden Durchfluss von 2-5 Liter/Minute. Sie sind entweder mit einer Trokar-Kanülen- oder Seldinger-Führungsdrahttechnik problemlos einzuführen, allerdings nur mäßig gut dirigierbar [50]. Weitlumige Drainagen aus PVC oder Silikon, etwa in einer Standarddimension von $24 \mathrm{~F}$, sollten wichtige Eigenschaften wie Transparenz, gute Verträglichkeit, Knickfestigkeit und aseptische bzw. antiadhäsive Oberflächenkonditionierung bei einer Länge $\leq 70 \mathrm{~cm}$ aufweisen. Sie werden entweder über eine Drainage-Stilett-, Trokar/Obturator-System (klassische Bülau-Technik) oder stumpfe, chirurgisch-digitale Präparation eingeführt und erlauben bereits beim Standardsog von $10 \mathrm{~cm} \mathrm{H}_{2} \mathrm{O}$ naturgemäß wesentlich höhere Durchflussraten von $10-15 \mathrm{l} / \mathrm{min}$ [50]. Damit bieten sie klare Vorteile, insbesondere bei großen Fisteln, bei Ergussbeteiligung und wenn eine Spül- oder Instillationstherapie (Pleurodese, Fibrinolyse) erwogen werden muss. Eine Gegenüberstellung der Präferenzkriterien verschiedener Drainagespezifikationen gibt Tab. 4.
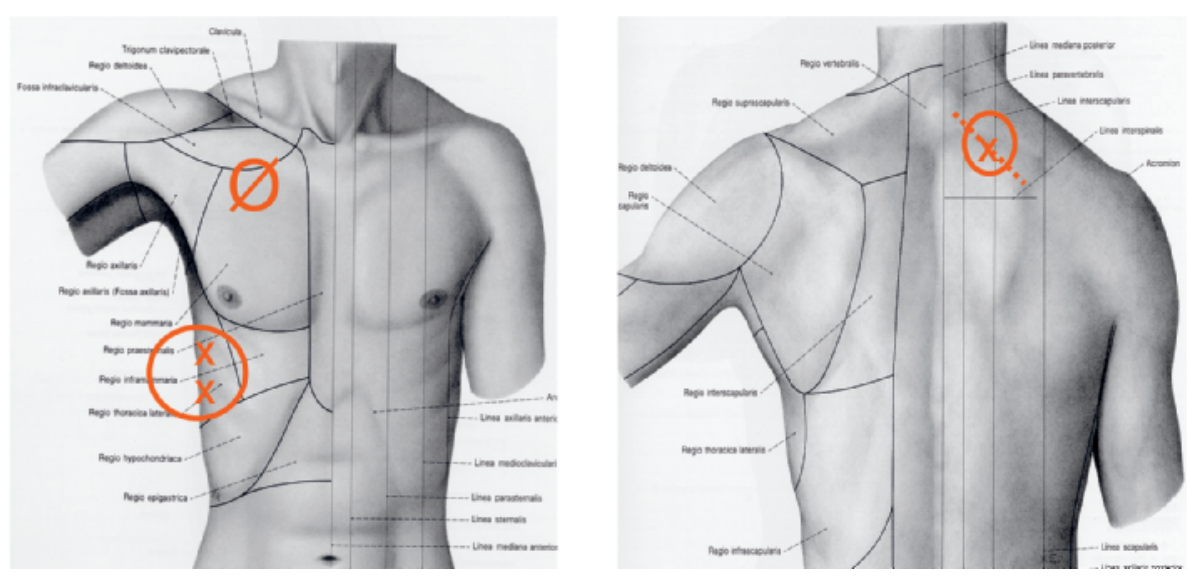

Abb. 2 Empfohlene und nicht empfehlenswerte Eingangsorte für Verweilkatheter und weitlumige Thoraxdrainagen. antero-basaler Zugang: XX

4. - 5. - 6. Interkostalraum mittlere bis vordere Axillarlinie.

$\varnothing$ apiko-frontaler Zugang (2. - 3. ICR medioklavik.) nur für einfache Aspiration, für Verweilkatheter und Drainagen ungeeignet. apiko-posteriorer Zugang: $X$

2. Interkostalraum paravertebral auf der halben Verbindungslinie von processus spinosus des 7. BWK mit dem angulus scapulae superior. 
Tab. 4 Drainagetherapie des Pneumothorax. Dünnlumige versus weitlumige Drainagen

\begin{tabular}{|c|c|c|}
\hline Material & Pros & Kontras \\
\hline $\begin{array}{l}\text { Katheter < } 16 \text { Ch } \\
\pm \text { Heimlich-Ventil }\end{array}$ & $\begin{array}{l}\text { geringer technischer Auf- } \\
\text { wand } \\
\text { geringer Pflegebedarf } \\
\text { gute Verträglichkeit } \\
\text { mobiler Patient }\end{array}$ & $\begin{array}{l}\text { Obturationsgefahr durch } \\
\text { Knicken u. Sekret begrenzte } \\
\text { Durchflussrate } \\
\text { keine Konditionierbarkeit } \\
\text { schlechte Dirigierbarkeit } \\
\text { bei Einlage } \\
\text { bei Sekret ungeeignet } \\
\text { pleurodeseinkompatibel }\end{array}$ \\
\hline $\begin{array}{l}\text { Drainagen } \\
>20 \mathrm{Ch} \\
\pm \text { Dauersog }\end{array}$ & $\begin{array}{l}\text { gute Dirigierbarkeit } \\
\text { optimale Funktion (hohe } \\
\text { Durchflussrate, geringe Ob- } \\
\text { turations- und Knickgefahr) } \\
\text { optimale Funktions- und } \\
\text { Durchgängigkeitskontrolle } \\
\text { bei Sekret gut geeignet } \\
\text { pleurodesekompatibel }\end{array}$ & $\begin{array}{l}\text { anspruchsvollere Einlage- } \\
\text { technik } \\
\text { höhere technische Anforde- } \\
\text { rungen und höherer Pflege- } \\
\text { bedarf } \\
\text { schlechtere Patientencom- } \\
\text { pliance und } \\
\text { eingeschränkte Mobilität }\end{array}$ \\
\hline
\end{tabular}

Drei kontrollierte Studien wurden zur Wirksamkeit der Pleuradrainage durchgeführt: eine Studie hat den Vorteil der kontinuierlichen Aspiration gegenüber einer einfachen Thoraxdrainage nicht beweisen können [51]. Eine andere Studie ergab eine Erfolgsquote von 93\% für die Pleuradrainage im Vergleich zu 67\% für die einfache Aspiration, wobei weder die Dauer des Krankenhausaufenthaltes noch die Rückfallquote in beiden Gruppen signifikant unterschiedlich waren [47]. In einer weiteren Studie [52] und einer Metaanalyse zufolge [53] erwiesen sich die klassische Thoraxdrainage und die Drainage in Verbindung mit einem Heimlich-Ventil bezüglich Komplikationen als gleichwertig, wobei letztere Technik allerdings eine ambulante Behandlung erlaubte und mit geringerer Schmerzreaktion verbunden war. Die zitierten drei kontrollierten Studien scheinen somit beim Pneumothorax die einfache Drainage oder einfache Aspiration $\mathrm{zu}$ favorisieren, wenn ausdehnungs- oder symptombedingt die Luftevakuierung des Pleuraraums zwingend ist. Nach einer neueren Studie genügt möglicherweise sogar die einfache Aspiration über eine Teflonkanüle [49], da zwischen Thoraxdrainage und einfacher Aspiration im Hinblick auf den Soforterfolg, Krankenhausverweilzeit und Rezidivhäufigkeit im Jahresverlauf kein Unterschied bestand, sich im Falle der einfachen Aspiration hingegen die Hospitalisierungsrate noch um die Hälfte verringerte.

\section{Rolle der Thorakoskopie}

Der in Lokalanästhesie durchgeführten internistischen Thorakoskopie (Pleuroskopie) kommt eine Schlüsselrolle im Management des Spontanpneumothorax zu, wenn eine trokar-gebundene weitlumige Drainagetherapie als Standardverfahren des evakuationspflichtigen Pneumothorax zugrunde gelegt wird. Mit nur geringfügig zusätzlichem logistischem Aufwand kann dann die Drainageeinlage zu einer zumindest orientierenden pleuralen Inspektion mit oft therapieentscheidenden Informationen genutzt werden. Die Vorteile dieses Vorgehens liegen auf der Hand und bestehen: (1) in einer sorgfältigen, auch dem CT überlegenen Lungenbeurteilung, (2) der Möglichkeit der direkten oder Fluoreszin-Autofluoreszenz-gestützten Leckagelokalisation mit ggf. Intervention zum Fistelschluss [54-56], (3) der optimalen Drainagepositionierung, (4) der ggf. sofortigen Durchführung
Tab. 5 Rolle der Thorakoskopie beim PTX Muster bzw. Klassifizierung endoskopischer Befunde und ihre Häufigkeitsverteilung (Historische Stadieneinteilung)*

\begin{tabular}{|c|c|c|c|}
\hline $\begin{array}{l}\text { Muster } \\
\text { (Typ) I } \\
40 \%\end{array}$ & $\begin{array}{l}\text { Muster } \\
\text { (Typ) II } \\
12 \%\end{array}$ & $\begin{array}{l}\text { Muster } \\
\text { (Typ) III } \\
31 \%\end{array}$ & $\begin{array}{l}\text { Muster } \\
\text { (Typ) IV } \\
17 \%\end{array}$ \\
\hline $\begin{array}{l}\text { „idiopathischer“ } \\
\text { PTX, normal er- } \\
\text { scheinende Lunge }\end{array}$ & $\begin{array}{l}\text { pleurale Adhäsionen, } \\
\text { dabei durch Einrisse } \\
\text { gehäuft } \\
\text { begleitender Häma- } \\
\text { tothorax }\end{array}$ & $\begin{array}{l}\text { Blasen u. Bullae } \\
<2 \mathrm{~cm} \varnothing\end{array}$ & $\begin{array}{l}\text { Bullae } \\
>2 \mathrm{~cm} \varnothing\end{array}$ \\
\hline
\end{tabular}

* modifiziert nach Swierenga/Vanderschueren Lung (1990) Suppl. 1122

der Talkpoudrage und (5) der Indikationsstellung zur primär chirurgischen Therapie bei entsprechend ausgedehnten Befunden $[55,57,58]$. Grundlage dieser Entscheidungen ist die Möglichkeit einer standardisierten Deskription und Typisierung prädisponierender Veränderungen. Swierenga und Vanderschueren schlugen hierfür 1990 eine Einteilung in 4 sog. „Stadien“ nach Tab. 5 vor [59]. Heute weiß man, dass dieses Konzept einer Stadiierung in progressive Schweregrade nicht haltbar ist, insbesondere aber, wie im Abschnitt „Pathophysiologie“ gezeigt, das „Stadium I“ der „intakten“ Lunge praktisch nicht existiert. Das Schema sollte daher lediglich zur Charakterisierung verschiedener „patterns“ oder Typen des Pneumothorax im Hinblick auf therapeutische Entscheidungen verwendet werden, wobei sich chirurgische Interventionen auf adhäsive und großbullöse Veränderungen des Typs II und IV konzentrieren sollten. Prägnante endoskopische Befunde zeigen Abb. 3-5.

Die derzeitigen Konsensempfehlungen europäischer wie amerikanischer Fachgesellschaften zum Akutmanagement des Spontanpneumothorax lassen sich so wie folgt zusammenfassen $[39,41,42]$ :

- Die alleinige Beobachtung ist gerechtfertigt beim Pneumothorax geringer Ausdehnung ( $<2 \mathrm{~cm}$ apikaler Pneuspalt) und/ oder wenn keine Symptome bestehen.

- Falls sich aufgrund der Ausdehnung und/oder Symptomatik eine Luftevakuierung als notwendig erweist, ist eine einfache Aspiration in ungefähr ${ }^{2} / 3$ der Fälle erfolgreich.

- Bei Misserfolg der einfachen Aspiration, ist eine Pleuradrainage zwingend, alternativ mit oder ohne Dauersog (d.h. mittels Heimlich-Ventil oder Wasserschloss) unter Abwarten des Spontanverschlusses des Luftlecks. Bei der Indikation zur Drainage erscheint unter Verzicht auf die Aspiration bereits primär die Versorgung über weitlumige Drainagen $(>20 \mathrm{Ch}$ ) als Therapie der Wahl aus Gründen der überlegenen Ergebnisse (bis zu 96\% Erfolgsrate), größeren Sicherheit und technischer Vorteile entsprechend Tab. 3. Die Einlage einer solchen Drainage erfolgt optimalerweise im Kontext einer Thorakoskopie (Pleuroskopie).

- Die Versorgung des Pneumothorax mit Spannungserscheinungen sollte gleichermaßen beim PSPTX und SSPTX, insbesondere beim ventilierten Patienten primär mittels weitlumiger Drainagen erfolgen. 

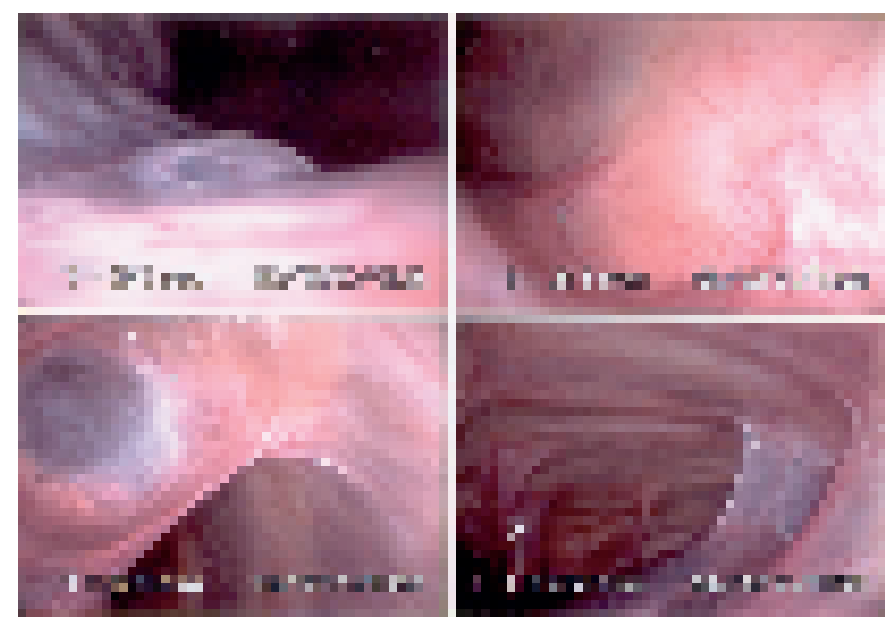

Abb. 3 PTX Muster (Typ) Il ausgedehnte Verwachsungsstränge.
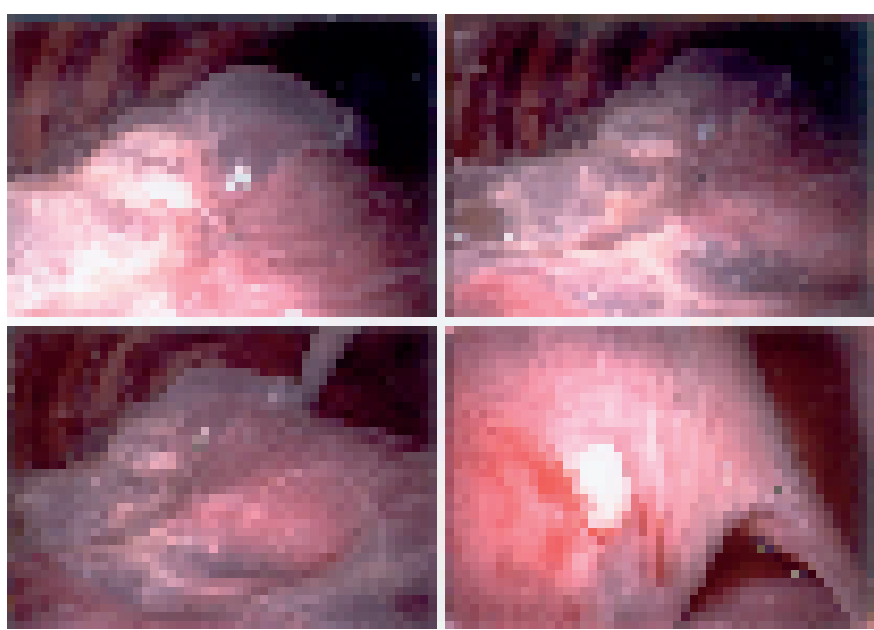

Abb. 5 PTX Muster (Typ) IV: zahlreiche Blasen $>2 \mathrm{~cm}$ und Bullae, vereinzelte Adhäsionen.

- Sekundäre Spontanpneumothoraxes sollten generell mit weitlumigen Drainagen versorgt werden, insbesondere in der Altersgruppe $>50$ Jahre und wenn Erguss jedweder Genese beteiligt ist.

\section{Prävention des Pneumothoraxrezidivs}

Präventive Strategien des Spontanpneumothorax gehen von der empirischen Rezidivhäufigkeit aus, die beim PSPTX etwa $30 \%$ beträgt (Spanne 13-54,2\%), nach dem ersten Rezidiv im Mittel aber bereits auf $62 \%$ ansteigt $[1,60]$. Noch wesentlich höher liegen die entsprechenden Raten beim SSPTX. Die Indikation zur Präventivtherapie ist somit spätestens beim 1. Rezidiv zu stellen. $\mathrm{Zu}$ den interventionellen Alternativen ergibt sich allerdings ein methodisches Problem: es gibt keine randomisierte und kontrollierte Studie, die eine internistische und eine chirurgische Vorgehensweise vergleicht, wie es vor einigen Jahren Janssen u. Mitarb. [20] hervorgehoben haben.

\section{Internistische Optionen}

Seit langem ist bekannt, dass eine Talkpleurodese durch Thorakoskopie (sog. „poudrage“ oder „talcage“) eines der wirksamsten

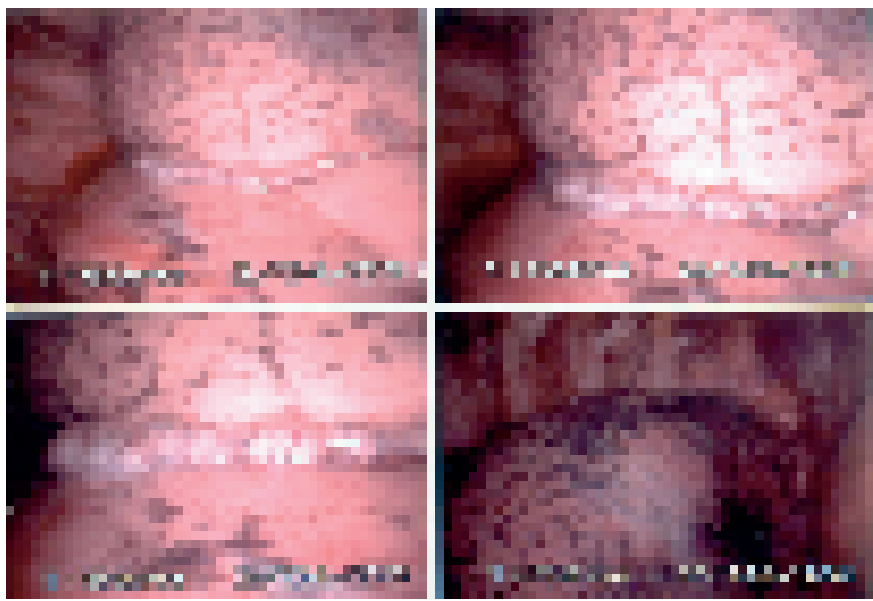

Abb. 4 PTX Muster (Typ) III: „Blebs“ und Blasen $<2 \mathrm{~cm}$ beim primären Spontanpneumothorax (charakteristischerweise am Lappenrand).
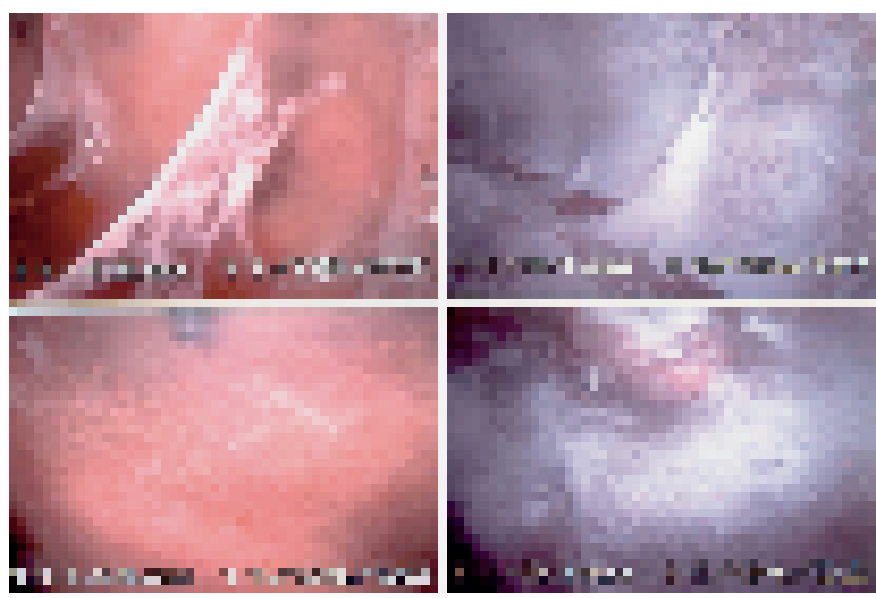

Abb. 6 Thorakoskopische Talk-Poudrage. Pleuraler Situs vor und nach Poudrage.

und auch kostengünstigsten Mittel der Pleurodese ist, und zwar aus klinischen [60] wie auch experimentellen Untersuchungen [61]. Die immer wieder aufflammende Kontroverse über die Risiken von Talk ist nicht gerechtfertigt, sofern man den Talk von Luzenac (Frankreich), der in Europa üblicherweise benutzt wird, verwendet $[62,63]$. Dies lässt sich durch Langzeitbeobachtungen belegen $[64,65]$. Die Risiken einer systemischen Talkdissemination, wie sie in einer neueren amerikanischen Studie [66] im Tierversuch beobachtet wurden, sind in Europa durch eine Studie, die dieses Protokoll wiederholt hat, nicht bestätigt worden [67]. Es darf vermutet werden, dass die Diskrepanz im Zusammenhang mit der nativen Partikelstruktur unterschiedlicher Talkpräparationen steht, da sich französischer Luzenac-Talk von dem amerikanischer Provenienz u. a. durch 3-fach größere Partikel unterscheidet [64]. Eine definitive klinische Einschätzung bleibt den Ergebnissen einer aktuell europaweit multizentrisch durchgeführten therapiebegleitenden Studie zur Überprüfung der Talk-Sicherheit („talc-safety-study“) vorbehalten [68]. Präliminäre Ergebnisse bestätigen bisher die hohe Sicherheit der französischen Talkpräparation. Das endoskopisch erzeugte Ergebnis der Talk-Poudrage zeigt Abb. 6. 
Zur Effektivität der Talkpleurodese hat die ESMEVAT-Gruppe (European Study on Medical Video-Assisted Thoracoscopy), eine randomisierte, multizentrische, europäische Studie durchgeführt [69] und die Rezidivraten nach einer einfachen Talkpuderung durch Thorakoskopie ( $2 \mathrm{~g}$ Talk) mit der einer konservativen Drainagebehandlung bei SPTX-Patienten verglichen: zwischen beiden Gruppen gab es keine signifikanten Unterschiede, was den Krankenhausaufenthalt, die Gesamtkosten, und die Schmerzen unter Verabreichung von Opiaten, betrifft. Nach 5 Jahren zeigte jedoch das Follow-up eine Rückfallquote des Pneumothorax von 34\% in der konventionell behandelten Gruppe, hingegen nur von $5 \%$ in der Gruppe, die mit einer einfachen Talkpleurodese durch Thorakoskopie $(p<0,01)$ behandelt worden war. Kaum weniger erfolgreich war in einer jüngeren prospektiven Studie [70] die Pleurodese mit Tetrazyklin-Hydrochlorid mit einer Rückfallrate von 9\% (6/66) in Bestätigung umfangreicher Daten bereits aus den 1970ger und 80ger Jahren [1,33,60]. Ein PSPTXPatient sollte deshalb wissen, dass sein Rückfallrisiko 6x höher ist, wenn die Behandlung konservativ mit einer Thoraxdrainage durchgeführt worden ist, auch wenn die meisten Patienten kein Rezidiv erleiden. Ein Patient mit einem PSPTX, bei dem ein Thoraxdrainage vorgesehen wird, sollte daher im Hinblick auf das Rezidivrisiko frei wählen können zwischen einer sofortigen symptomatischen Behandlung, die wenig Unannehmlichkeit mit sich bringt aber die Rezidivgefahr offen lässt, und einer wie auch immer gearteten präventiven Intervention, die ein ipsilaterales Rezidiv mit hoher Wahrscheinlichkeit verhindert.

\section{Chirurgische Optionen}

Die Ziele der chirurgischen Therapie des SPTX sind einerseits die Ablation von großbullösen Lungenveränderungen bzw. die Parenchymresektion mit Fistelverschluss und die Lösung von Adhäsionen, andererseits die Durchführung der Pleurodese. Für letztere Option bieten sich wahlweise die Talkpoudrage (analog zur internistischen Technik), diverse Abrasionstechniken sowie die partielle Pleurektomie an. Alle chirurgischen Interventionen können prinzipiell über einen traditionellen Zugang mit formeller Thorakotomie (ggf. auch Mini-Thorakotomie) oder über eine minimal-invasive videoassistierte Technik (VATS) durchgeführt werden. Die technische Perfektion der VATS ist heute soweit entwickelt, dass sie mit sämtlichen Operationsverfahren im Sinne der Bullaablation und Parenchymresektion mittels automatischer Klammernahtgeräten (sog. „Staplers“) oder auch Elektrokauterisierung bzw. Laserablation voll kompatibel ist. Die Konversationsrate zur formellen Thorakotomie beträgt dabei weniger als 9\% [71]. Dies gilt gleichermaßen für die klassische Pleurektomie und diverse neuere Pleurodesetechniken (Talk-Poudrage, Skarifizierung, Trockengazeabrasion, Endoabrader, Argonplasmabeamer, Laserkoagulation) [71 - 74]. Den Operationssitus bei der klassischen Pleurektomie zeigt Abb. 7. Vielfach wird heute bei der VATS-Intervention der Neodym-YAG-Laser (MY 40 1.3) in der lungenspezifischen „bare fiber“-Spezifikation verwendet, da diese vielseitige Technik sich gleichermaßen für die Bullaablation, die Stranglösung wie die photothermische Pleurodese eignet [71].

Damit hat sich die VATS zum führenden Ansatz für alle chirurgischen Optionen im Management des Pneumothorax entwickelt [71-74]. Diese Präferenz ist möglicherweise (zumindest beim SSPTX) nicht gerechtfertigt, wenn man zwei randomisierte Ver-
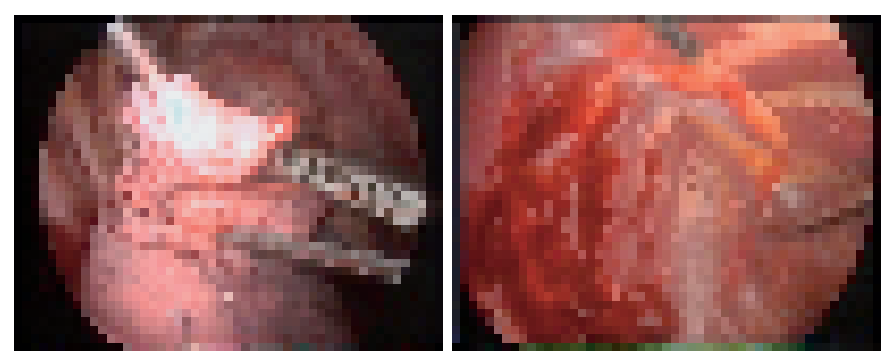

Abb. 7 VATS-Präparation bei der Bullaablation und der partiellen Pleurektomie.

gleiche zwischen VATS-Techniken und konventioneller Thorakotomie zugrunde legt, in welchen bei längerer Operationsdauer die VATS-Versagerquote mit $13 \%$ versus $3,3 \%$ bzw. $8 \%$ versus $2 \%$ signifikant höher lag $[73,74]$. Es gibt zahlreiche weitere, nicht randomisierte Studien über chirurgische Verfahren in der Behandlung des Spontanpneumothorax sei es durch VATS, Thorakotomie, Mini-Thorakotomie mit oder ohne Bullektomie bzw. Parenchymresektion. Die Ergebnisse variieren erheblich und oft fehlt ein Follow-up oder es ist ungenügend, so dass hier kein einheitliches Bild resultiert [75-83].

Auch innerhalb der chirurgisch-technischen Pleurodeseoptionen gibt es deutliche Unterschiede, wobei die partielle Pleurektomie und die "talcage“ mit einer Versagerquote von lediglich $2 \%$ bzw. $5 \%$ die Spitzenpositionen einnehmen und als annähernd äquipotente Goldstandardtechniken angesehen werden dürfen $[50,56]$. Insbesondere beim SSPTX ist bei den verschiedenen Abrasionstechniken immerhin mit einer Versagerquote bis zu 12,5\% zu rechnen [84]. In der konkreten Erwägung einer Talkpleurodese (gleich ob chirurgisch oder internistisch) versus eine partielle Pleurektomie wurde von chirurgischer Seite beim jugendlichen Patienten der Einwand vorgebracht, dass bei der „talcage“ durch die Intensität der pleuralen Syndesmose chirurgische Lungeneingriffe aus anderweitiger Indikation prospektiv massiv erschwert sein können [85]. Ähnlich wurde kürzlich auch aus der Sicht der Organtransplantologie argumentiert [86]. Diese durchaus bedenkenswerten Gesichtspunkte stellen allerdings weder unter Effektivitäts- noch Kostenaspekten ein Argument gegen die Talkpleurodese dar.

Beim PSPTX sollte die Entscheidung über die optimale Präventivstrategie ausgehend von den beschriebenen pathophysiologischen Befunden nicht vom videothorakoskopischen oder CT-gestützten Nachweis von „blebs“ oder ELCs abhängig gemacht werden. Die Behandlung muss vielmehr unabhängig davon durch eine pleurodetische Maßnahme so effektiv wie nur möglich gestaltet werden. So haben Schramel u. Mitarb. [87] in einer retrospektiven Studie nachgewiesen, dass beim PSPTX eine aggressive, chirurgische Therapie durch VATS wirksamer ist als eine konservative, einfache Drainagebehandlung. Hierbei wurde eine Gruppe von 112 primär konservativ mittels Drainagetherapie versorgter Patienten, bei denen erst bei Komplikationen oder Versagen chirurgisch interveniert wurde, mit 97 primär durch VATS versorgten Patienten verglichen. In der Kostenanalyse (Tab.6) mussten die Autoren allerdings zunächst einräumen, dass die prozeduralen Kosten dieser Behandlung um $62 \%$ hätten verringert werden können, wenn sie eine einfache Talkpleurode- 
Tab. 6 Konservative versus interventionelle Strategien beim Spontanpneumothorax. Analyse unter Kostengesichtspunkten

\begin{tabular}{|llll}
\hline Kostenfaktor & $\begin{array}{l}\text { Periode I, } \mathbf{n = 1 1 2} \\
\text { primär Drain, VATS } \\
\text { bei Versagen } \\
\text { Währungseinheiten }\end{array}$ & $\begin{array}{l}\text { Periode II, } \boldsymbol{n}=\mathbf{9 7} \\
\text { primär VATS }\end{array}$ & W-Wert \\
\hline Primärtherapie & $586 \pm 182$ & $2403 \pm 805$ & $<0,0001$ \\
\hline Klinikverweilzeit & $9620 \pm 7849$ & $7926 \pm 3006$ & 0,03 \\
\hline Komplikationen & $872 \pm 11165$ & $91 \pm 395$ & $<0,0001$ \\
\hline subtotal & $11087 \pm 7275$ & $10420 \pm 3452$ & $\mathrm{NS}$ \\
\hline Wartezeit & $-588 \pm 2239$ & $-4558 \pm 3013$ & $<0,0001$ \\
\hline Gesamtkosten & $10499 \pm 7723$ & $5862 \pm 3013$ & $<0,0001$ \\
\hline
\end{tabular}

FMNH Schramel, TG Sutedja, JCE Braber u. Mitarb. ERJ (1996) 9:1821 - 1825

se durch Thorakoskopie, anstatt eine technisch aufwendige und teure VATS, durchgeführt hätten [88]. In der weiteren Analyse deutete sich jedoch unter der Prämisse einer signifikanten Verkürzung der langen präoperativen klinischen Wartezeit der VATS-Strategie eine deutliche Kostenreduktion an.

Zusammengefasst lässt die derzeitige Datenlage keine zwingende Schlussfolgerung zu, welchem Verfahren in der Präventivtherapie des PSPTX ergebnisbezogen der Vorzug zu geben ist. Die ESMEVAT-Ergebnisse (European Study on Medical Videoassisted-Thoracoscopy) [69] belegen, dass die internistisch thorakoskopische "talcage" eine hocheffektive, einfache, sichere und auch in der Schmerzkontrolle gut handhabbare minimal-invasive Methode der Pleurodese ist, die in der Kostenanalyse klare Vorteile gegenüber der VATS-Technik aufweist. In den meisten Zentren dürfte derzeit die Abwägung zwischen den internistischen und VATS-gestützten Therapieverfahren eine Ermessensentscheidung bleiben, in die ortsabhängige Einschätzungen und logistische Faktoren wie anästhesiologische Ressourcen, Patientenwunsch, Präferenz für Einloch- oder Mehrlochtechnik und unterschiedliche chirurgisch-internistische Absprachen eingehen. Wenn die Vorteile der Pleuroskopie-gestützten Behandlung der akuten SPTX-Episode genutzt werden, ergibt sich als Leitlinie für das Gesamtmanagement das Schema der Abb. $8[55,58]$.

\section{Besonderheiten der Therapie des SSPTX}

Die Therapie des SSPTX kann von der des PSPTX sowohl in der Akutbehandlung als auch in der Rezidivprävention differieren. Die oft massiven destruktiven Lungenveränderungen, Adhäsionen, Kammerungen und Leckagen mit konsekutivem schwer beherrschbarem Weichteil- und Mediastinalemphysemen können bereits in der Akutversorgung entweder ein komplexes Mehrfach-Drainageregime oder chirurgische Maßnahmen erzwingen. $\mathrm{Zu}$ den chirurgischen Indikationen im Akut-Management des komplizierten SSPTX, selten auch des PSPTX, zählen daher $[41,42,48]$ :

- über > 7 Tage persistierende Parenchymfisteln,

- der Spontanhämatopneumothorax,

- das trotz Drainagetherapie progrediente und hämodynamisch oder respiratorisch wirksame Mediastinalemphysem,

- der (seltene) bilaterale SPTX.

Entsprechend der hohen Rezidivrate sollte auch beim SSPTX in jedem Fall bereits das Erstereignis Veranlassung zur Durchführung einer Präventivtherapie geben. Grundsätzlich ergibt sich auch hierbei die Abwägung zwischen dem internistischen und chirurgischen Vorgehen. Es gibt jedoch valide Daten, die gezeigt haben, dass auch beim fortgeschrittenen Emphysem mit entsprechend großbullösen Veränderungen und selbst beim Vorliegen einer respiratorischen Insuffizienz die internistisch-thorakoskopische Talkpleurodese ohne schwerwiegende Komplikationen technisch gut durchführbar ist, und die Ergebnisse denen der chirurgischen Verfahren nicht nachstehen $[89,90]$. So erwies sich in einer prospektiven Studie zur Wirksamkeit der internistisch-thorakoskopischen Talk-Poudrage beim SSPTX infolge fortgeschrittener COPD ( $\mathrm{FEV}_{1} 41 \pm 14 \%$ Soll) diese zu $95 \%$ erfolgreich bei einer akzeptablen Mortalität von 10\% [90]. Dies ist umso bedeutsamer, als in den klinisch häufigen Situationen fortgeschrittenen Lebensalters, bei massivem generalisiertem Emphysem, schlechtem Allgemeinzustand, interstitiellen Lungenkrankheiten und Multimorbidität chirurgische Optionen sowohl an technische als auch anästhesiologische Grenzen der Durchführbar-

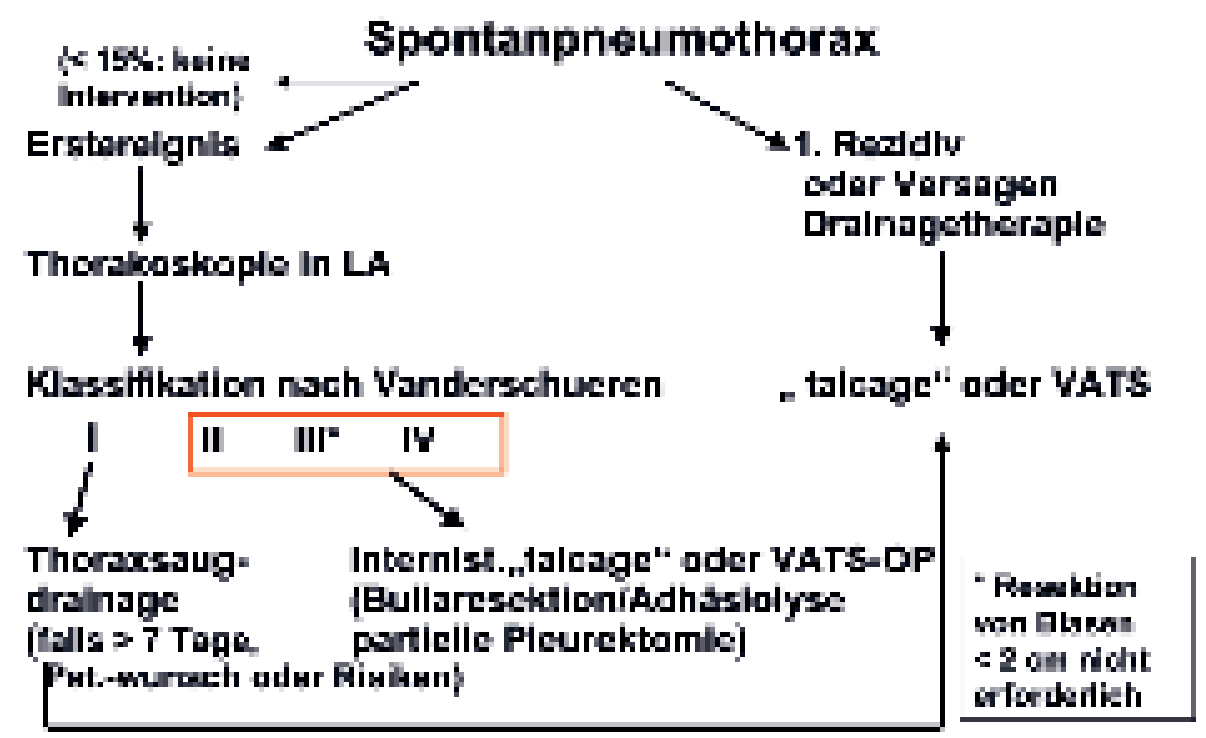

Abb. 8 Thorakoskopie-basierter Therapiealgorithmus. 
keit mit unvertretbarer Mortalität stoßen. Es ergibt sich so in diesen Fällen eine klare Präferenz internistischer Pleurodesetechniken mittels Tetrazyklin oder Talk.

\section{Respirator-assoziierter Pneumothorax (RAPTX)}

Der respiratorassoziierte PTX entsteht meist multifaktoriell und schließt in seiner Genese traumatische Ursachen wie vena subclavia-Fehlpunktion, Thorakozentese, transbronchiale Biopsie und atemmechanisches Barotrauma ebenso ein wie präexistente Lungenveränderungen im Sinne des sekundären Spontanpneus etwa bei COPD, interstitiellen Lungenerkrankungen und Pneumonie. Insbesondere ARDS und die opportunistische Pneumocystis-Pneumonie können in bis zu 60\% durch einen PTX kompliziert sein; beidseitige Pneus sind nicht ungewöhnlich [91]. Innerhalb eines gegebenen intensivmedizinischen Rahmens ist beim beatmeten Patienten der durchschnittliche Atemwegsdruck die wichtigste Determinante des Pneumothorax-Risikos. Eine PEEP-Beatmung bis $15 \mathrm{~cm} \mathrm{H}_{2} \mathrm{O}$ ist global mit 4\% Pneumothoraxrisiko verbunden, jede weitere Erhöhung steigert das Risiko um das 2-4fache [91]. Eine besonders enge Korrelation scheint zum Gradienten zwischen end-inspiratorischem Plateaudruck und PEEP-Level zu bestehen, der für den maximalen Inflationsgrad der Lunge verantwortlich ist [92]. Insofern ist es richtiger, von einem „Volumentrauma“ als von einem „Barotrauma“ zu sprechen. Alle Strategien zur Prävention bzw. Beherrschung des RAP konzentrieren sich daher auf Maßnahmen zur Atemwegsdruck- bzw. Atemminutenvolumenreduktion im Sinne der „permissiven Hypoventilation“, wie sie in Tab. 7 zusammengefasst sind. Die Drainagetherapie beim ventilierten Patienten sollte ausnahmslos mittels weitlumiger Drainagen erfolgen, bleibt aber auch dann noch komplikationsreich. Die Beherrschung großer bronchopleuraler Fisteln oder des Mediastinalemphysems unter Beatmungsbedingungen gehört zu den schwierigsten intensivmedizinischen Herausforderungen und kann auch unter Aufbietung komplexer Strategien wie der JetVentilation, seitengetrennter bzw. einseitiger Beatmung, multiplen Drainagen, Pleurodeseversuchen und interventionellem Fistelverschluss den Arzt vor kaum lösbare Aufgaben stellen (Tab. 7).

\section{Praktische Schlussfolgerungen}

1. Ein primärer, asymptomatischer Spontanpneumothorax sowie ein Pneumothorax geringer Ausdehnung benötigt keine interventionelle Behandlung.

2. Beim Erstereignis des interventionspflichtigen primären Spontanpneumothorax kann eine einfache Aspiration in ca. $2 / 3$ der Fälle mit Erfolg durchgeführt werden.

3. Im Fall eines Misserfolges der Aspiration kann ein kleinkalibriger Drain $(8-16 \mathrm{~F})$ mit oder ohne Dauersog erwogen werden. Optimal ist eine weitlumige, in Verbindung mit der Thorakoskopie anzulegende Drainage $(\geq 20 \mathrm{~F})$ mit gleichzeitiger Evaluierung prädisponierender Lungen-/Pleuraveränderungen.
Tab. 7 Management des ventilatorassoziierten Pneumothorax

Barotrauma-Präventivstrategien

- interstitielle Lungenerkrankungen (ARDS u. a.)

- BiPAP oder CPAP/PCV statt VCV-Centilation

- Inverse-Ratio-Ventilation mit Paw max. $<35$ und PEEP $<15 \mathrm{~cm} \mathrm{H}_{2} \mathrm{O}$

- permissive Hypoventilation mit Atemzugvolumen $<6 \mathrm{ml} / \mathrm{kg} \mathrm{KC}$

- obstruktive Atemwegserkrankungen

- niedriges AMV (permissive Beatmung)

- kein oder geringer PEEP

Erkennung des PTX

- abrupter Paw max-Anstieg

- abrupte Gasaustauschstörung

Behandlungsoptionen bei bronchopleuraler Fistel

- weitlumige Drainagen (>24 Ch)

- Seitlagerung: kranke Seite abhängig

- Registrierung des Fisteldurchflusses = inspirat. minus exspirat. AMV

- Minimierung des Durchflusses durch niedrige Drainagensogeinstellung (bis „Null“-Sog) und Beatmungsmustermodifikation (ASB-Modus)

- Versuch: interne bronchoskop. Fistelokklusion (Fibrinkleber)

- Versuch: externer Fistelschluss (Talkuminstillation)

- Hochfrequenz Jet-Beatmung (nicht gesichert)

- Doppellumenintubation mit einseitiger oder seitengetrennter Beatmung

- ultimativ: chirurgische Intervention falls kompatibel mit klinischem Zustand

$\mathrm{PCV}=$ pressure control ventilation, $\mathrm{VCV}=$ volume control ventilation, ASB = augmentierte Spontanatmung

4. Die Durchführung einer Pleurodese sollte in Absprache mit dem Patienten (insbesondere bei beruflichen Risiken) bereits beim Erstereignis eines primären Spontanpneumothorax in Betracht gezogen werden. Jeder Patient mit einem Spontanpneumothorax bedarf ggf. dringend der Raucherentwöhnung!

5. Die gegenwärtige Datenlage liefert erfolgsbezogen keine eindeutigen Präferenzkriterien im Entscheid zwischen internistischer Thorakoskopie und einem chirurgischen Vorgehen. Hierzu bedarf es neuer randomisierter und kontrollierter Studien.

6. In der Prävention eines Rezidivs des primären Spontanpneumothorax kann die internistische Pleurodese oder die partielle Pleurektomie das Rückfallrisiko wesentlich verringern. Die einfache Talkpleurodese ohne Bullektomie bzw. Parenchymresektion scheint genauso wirksam wie die Pleurodese in Kombination mit Lungenresektion. Die superfizielle parietoviszerale Pleurodese ist naheliegenderweise auch als Präventivtherapie der Wahl beim SPTX-Rezidiv anzusehen.

7. Die Versorgung des komplikationsreichen sekundären Spontanpneumothorax und respiratorassoziierten PTX kann komplexe internistische und chirurgische Strategien erfordern mit oder ohne Parenchymresektion und multiplen Drains, in jedem Falle aber Durchführung der Pleurodese.

8. Der respiratorassoziierte PTX stellt eine Mischform von traumatischem und sekundärem Spontanpneumothorax dar. Er bedarf primär der Versorgung mit weitlumigen Drainagen und bei Komplikationen oft multipler Interventionen. Zur Prävention und auch zur Beherrschung ist die sorgfältige Beachtung drucksenkender Beatmungsprotokolle essentiell. 
Abkürzungen

PTX $=$ PneumoThoraX

SPTX = SpontanPneumoThoraX

PSPTX = Primärer SpontanPneumoThoraX

SSPTX = Sekundärer SpontanPneumoThoraX

RAPTX $=$ RespiratorAssoziierter PneumoThoraX

ELC $=$ „Emphsema Like Changes“

VATS $=$ VideoAssisted Thoracic Surgery

\section{Literatur}

${ }^{1}$ Light RW. Management of spontaneous pneumothorax. Am Rev Respir Dis 1992; 148: 245-248

2 Miller AC, Harvey JE, BTS oboSoCC. Guidelines for the management of spontaneous pneumothorax. BMJ 1993; 307: 114-116

${ }^{3}$ Henry M, Arnold T, Harvey J. BTS guidelines for the management of spontaneous pneumothorax. Thorax 2003; 58 (Supplement): 39-52

${ }^{4}$ Gupta D, Hansell A, Nichols T et al. Epidemiology of pneumothorax in England. Thorax 2000; 55: 666-671

${ }^{5}$ Melton LJ, Hepper NCG, Offord KP. Incidence of spontaneous pneumothorax in Olmsted County, Minnesora: 1950 - 1974. Am Rev Respir Dis 1979; 29: 1379-1382

${ }^{6}$ Bense L, Eklund G, Wiman LG. Smoking and the increased risk of contracting spontaneous pneumothorax. Chest 1987; 92: 1009-1012

${ }^{7}$ Bense L, Wiman LG, Hedenstierna G. Onset of symptoms in spontaneous pneumothorax: correlations to physical activity. Eur J Respir Dis 1987; 71 : $181-186$ (III)

${ }^{8}$ Noppen M, Verbanck S, Harvey J et al. Music: a new cause of primary spontaneous pneumothorax. Thorax 2004; 59: 772 - 724

${ }^{9}$ Jansveld CA, Dijkman JH. Primary spontaneous pneumothorax and smoking. BMJ 1975; 4: 559-560

${ }^{10}$ Schramel FM, Golding RP, Haakman CD et al. Expiratory chest radiographs do not improve visibility of small apical pneumothoraces by enhanced contrast. Eur J Respir Dis 1996; 9: 406-409

${ }^{11}$ Glazer H, Anderson DJ, Wilson BS et al. Pneumothorax: appearances on lateral chest radiographs. Radiology 1989; 173: 707-711

12 Bourgoin P, Cousineau G, Lemire P et al. Computed tomography used to exclude pneumothorax in bullous lung disease. J Can Ass Radiol 1985; 36 : $341-342$

13 Sattler A. Zur Behandlung der Spontanpneumothorax mit besonderer Berücksichtigung der Thorakoskopie. Beitr Klin Tuberk 1937; 89: $394-408$

${ }^{14}$ Mitlehner W, Friedrich M, Dissmann W. Value of computer tomography in the detection of bullae and blebs in patients with primary spontaneous pneumothorax. Respiration 1992; 59: 221 - 227

${ }^{15}$ Lesur O, Delorme N, Fromaget JM et al. Computed tomography in the etiologic assessment of idiopathic spontaneous pneumothorax. Chest 1990; 98 : $341-347$

${ }^{16}$ Baronofsky ID, Warden HG, Kaufmann JL et al. Bilateral therapy for unilateral spontaneous pneumothorax. J Thorac Surg 1957; 34: $310-322$

17 Ikeda M, Uno A, Yamane Y et al. Median sternotomy with bilateral bullous resection for unilateral spontaneous pneumothorax, with special reference to operative indications. J Thorax Cardiovasc Surg 1988; 96: $615-620$

${ }^{18}$ Radomsky J, Becker HP, Hartel W. Pleuroporosität beim idiopatischen Spontanpneumothorax. Pneumologie 1989; 43: 250-253

${ }^{19}$ Ohata M, Suzuki H. Pathogenesis of spontaneous pneumothorax: with special reference to the ultrastructure of emphysematous bullae. Chest 1980; 77: 771-776

20 Janssen JP, Schramel FMNH, Sutedja TG et al. Videothoracoscopic appearance of first and recurrent pneumothorax. Chest 1995; 108: $330-334$

${ }^{21}$ Bense L, Lewander R, Eklund G et al. Nonsmoking, non-alpha-1-antitrypsin deficiency-induced emphysema in non-smokers with healed spontaneous pneumothorax, identified by computed tomography of the lungs. Chest 1993; 103: 433-438
${ }^{22}$ Schramel FMNH, Meyer CLM, Postmus PE. Inflammation as cause of spontaneous and emphysematous-like changes: results of bronchoalveolar lavage. Eur Respir J 1995; 19: 397s

${ }^{23}$ Schramel FMNH. Current aspects of spontaneous pneumothorax. Eur Respir J 1997; 10: 1372-1379

${ }^{24}$ Sahn SA, Heffner JE. Spontaneous pneumothorax. N Engl J Med 2000 342: $868-874$

25 Baumann MH. Do blebs cause primary spontaneous pneumothorax? pro: blebs do cause primary spontaneous pneumothorax. J Bronchol 2002; 9: $313-18$

${ }^{26}$ Noppen M. Do blebs cause primary spontaneous pneumothorax? Con: Blebs do not cause primary spontaneous pneumothorax. J Bronchol 2002; 9: 319-23

${ }^{27}$ Horio H, Nomori H, Kobayaski R et al. Impact of additional pleurodesis in video-assisted thoracoscopic bullectomy for primary spontaneous pneumothorax. Surg Endosc 2002; 16: 630-634

${ }^{28}$ Loubani M, Lynch V. Video-assisted thoracoscopic bullectomy and acromycin pleurodesis: an effective treatment for spontaneous pneumothorax. Resp Med 2000; 94: 888-890

${ }^{29}$ Noppen M, Baumann MH. Pathogenesis and treatment of primary spontaneous pneumothorax: an overview. Respiration 2003; 70: $431-438$

${ }^{30}$ Hatz RA, Kaps MF, Meimarakis G et al. Long-term results after videoassisted thoracoscopic surgery for wedge resection without pleurodesis or pleurectomy. Eur J Cardio-thorac Surg 2000; 10: 656-659

${ }^{31}$ Dines DE, Clagett OT, Payne WS. Spontaneous pneumothorax in emphysema. Mayo Clin Proc 1970; 45: $481-487$

32 Tanaka F, Itoh M, Esaki H et al. Secondary spontaneous pneumothorax. Ann Thorac Surg 1993; 55: 372 - 376

${ }^{33}$ Tunon-de-Lara JM, Constans J, Vincent MP et al. Spontaneous pneumothorax associated with Pneumocystis carinii pneumonia. Chest 1992; 101: 1177 - 1178

${ }^{34}$ Light RW, O'hara VS, Moritz TE et al. Intrapleural tetracycline for the prevention of recurrent spontaneous pneumothorax: results of a Department of Veteran Affairs cooperative study. JAMA 1990; 264: $2224-2230$

${ }^{35}$ Lippert HL, Lund O, Blegvad S et al. Independent risk factors for cumulative recurrence rate after first spontaneous pneumothorax. Eur Respir J 1991; 4: 324-331

${ }^{36}$ British Thoracic Society Research Committee. Comparison of simple aspiration with intercostal drainage in the management of spontaneous pneumothorax. Thorax 1993; 48: 430-431

${ }^{37}$ Edenborough FB, Hussain I, Stableforth DE. Use of a Heimlich flutter valve for pneumothorax in cystic fibrosis. Thorax 1994; 49: $1178-1179$

38 Baumann MH, Strange C. The clinician's perspective on pneumothorax management. Chest 1997; 112: $822-828$

39 Janssen JP, Cuesta MA, Postmus PE. Behandeling van spontane pneumothorax: enquete onder Nederlandse longartsen. Ned Tijdschr Geneesk 1994; 138: 661-664

${ }^{40}$ Kinasewitz GT. Pneumothorax. Sem Respir Crit Care Med 1995; 16 (4): 293-302

${ }^{41}$ Baumann MH, Strange C, Heffner JE et al. Management of spontaneous pneumothorax. An American College of Chest Physicians Delphi Consensus Statement. Chest 2001; 119: 590-602

${ }^{42}$ Davies RJO, Gleeson FV, Ali N et al. BTS guidelines for the management of pleural disease. Thorax 2003; 58 (Suppl. II): ii39-ii52

${ }^{43}$ Archer GJ, Hamilton AAD, Upadhyag R et al. Results of simple aspiration of pneumothoraces. Brit J Dis Chest 1985; 79: 177- 182

${ }^{44}$ Spencer-Jones J. A place for aspiration in the treatment of spontaneous pneumothorax. Thorax 1985; 40: 66-67

${ }^{45}$ Harvey J. Simple aspiration vs. intercostal tube drainage for spontaneous pneumothorax in patients with normal lungs. BMI 1994; 309: $1338-1339$

${ }^{46} \mathrm{Ng}$ AWK, Chan KW, Lee SK. Simple aspiration of pneumothorax. Singapore Med J 1994; 35: 50-52

47 Andrivet P, Djedaim K, Teboul JL et al. Spontaneous pneumothorax, comparison of thoracic drainage vs. immediate or delayed needle aspiration. Chest 1995; 108: 335-340

${ }^{48}$ Dines DE, Claget OTI, Payne WS. Spontaneous pneumothorax in emphysema. Mayo Clin Proc 1970; 45: 481 - 487

${ }^{49}$ Noppen M, Alexander P, Driesen P et al. Manual aspiration versus chest tube drainage in first episodes of primary spontaneous pneumothorax. A multicenter, prospective, randomized pilot study. Am J Respir Crit Care Med 2002; 165: 1240-1244 
${ }^{50}$ Baumann $\mathrm{MH}$, Strange $\mathrm{CH}$. Treatment of spontaneous pneumothorax. A more aggressive approach? Chest 1997; 112: 789-804

${ }^{51}$ So SY, Yu DY. Catheter drainage of spontaneous pneumothorax: suction or no suction, early or late removal. Thorax 1982; 38: 46-48

52 Röggla M, Wagner A, Brunner C et al. The management of pneumothorax with the thoracic vent versus conventional intercostal tube drainage. Wien Klin Wochschr 1996; 108: 330-333

${ }^{53}$ Devenand A, Koh MS, Ong TH et al. Simple aspiration versus chest tube insertion in the management of primary spontaneous pneumothorax: a systematic review. Respiratory Medicine 2004; 98: 579-590

${ }^{54}$ Noppen M, Stratakos G, Verbanck S et al. Fluorescein-enhanced autofluorescence thoracoscopy in primary spontaneous pneumothorax. Am J Respir Crit Care Med 2004; 170: 680-682

${ }^{55}$ Boutin C, Astoul P, Rey F et al. Thoracoscopy in the diagnosis of spontaneous pneumothorax. Clin Chest Med 1995; 16/3: 497-503

${ }^{56}$ Takeno Y. Thoracoscopic treatment of spontaneous pneumothorax. Ann Thorac Surg 1993; 56: 688-90

${ }^{57}$ Loddenkemper R, Boutin C. Thoracoscopy: present diagnostic and therapeutic indications. Eur Respir J 1993; 5: 1544- 1555

${ }^{58}$ Frank W. Pneumothorax: Akutversorgung und Techniken der Pleurodese. Atemw.-Lungenkrkh 1998; 24/10: $452-460$

${ }^{59}$ Vanderschueren RG. The role of thoracoscopy in the evaluation and management of pneumothorax. Lung 1990; (Suppl.): 1122-1125

${ }^{60}$ Sadikot RT, Greene T, Meadows K et al. Recurrence of primary spontaneous pneumothorax. Thorax 1997; 52: 805-809

${ }^{61}$ Bresticker MA, Oba J, LoCicero J et al. Optimal pleurodesis: a comparison study. Ann Thorac Surg 1993; 55: $364-367$

${ }^{62}$ Lange P, Mortensen J, Groth S. Lung function $22-35$ years after treatment of idiopathic spontaneous pneumothorax with talc poudrage or simple drainage. Thorax 1988; 43: 559-561

63 el Khawand C, Marchandise FX, Mayne A et al. Spontaneous pneumothorax. Results of pleural talc therapy using thoracoscopy. Rev Mal Respir 1995; 12 (3): 275-281

${ }^{64}$ Light RW. Pro/Con editorials. Talc should not be used for pleurodesis. Am J Respir Crit Care Med 2000; 162: 2024-2026

${ }^{65}$ Sahn SA. Pro/Con editorials. Talc should be used for pleurodesis. Am J Respir Crit Care Med 2000; 162: 2023-2024

${ }^{66}$ Werebe EC, Pazetti R, Milanez de Campos JR et al. Systemic distribution of talc after intrapleural administration in rats. Chest 1999; 115: $190-193$

${ }^{67}$ Fraticelli A, Robaglia-Schlupp A, Riera $\mathrm{H}$ et al. Distribution of calibrated talc after intrapleural administration: an experimental study in rats. Chest 2002; 122: $1737-1741$

68 Janssen JP, Noppen M, Astoul P et al. persönliche Mitteilung

${ }^{69}$ Tschopp JM, Boutin C, Astoul P et al. Talcage by medical thoracoscopy for primary spontaneous pneumothorax is more cost-effective than drainage: a randomised study. Eur Respir J 2002; 20: 1003-1009

${ }^{70}$ Alfageme I, Moreno L, Huertas C et al. Spontaneous pneumothorax: long term results with tetracycline pleurodesis. Chest 1994; 106: $347-350$

${ }^{71}$ Passlick B, Born C, Thetter O. Ergebnisse der minimal invasiven Chirurgie (MIC) bei der operativen behandlung des rezidivierenden oder persistierenden primären Spontanpneumothorax. Pneumologie 1997; 51: $1135-1139$

72 Inderbitzi RGC, Furrer M, Striffeler H et al. Thoracoscopic pleurectomy for treatment of complicated spontaneous pneumothorax. J Thorac Cardiovasc Surg 1993; 105/1: $84-88$

${ }^{73}$ Waller DA, Forty J, Moritt GN. Video-assisted thoracoscopic surgery versus thoracotomy for spontaneous pneumothorax. Ann Thorac Surg 1994; 58: $372-376$

${ }^{74}$ Sekine Y, Miyato Y, Yamada H et al. Video-assisted thoracoscopic surgery does not deteriorate postoperative pulmonary gas exchange in spontaneous pneumothorax patients. Eur J Cardiothorac Surg 1999; 16: $48-53$
${ }^{75}$ Bernard A, Belichard C, Goudet P et al. Pneumothorax spontanée. Comparaison de la thoracoscopie et de la thoracotomie. Rev Mal Resp 1993; 10: $433-436$

${ }^{76}$ Yim AP, Liu HP. Video assisted thoracoscopic management of primary spontaneous pneumothorax. Surg Laparosc Endosc 1997; 7: 236-240

77 Liu HP, Chang CH, Lin PJ et al. Thoracoscopic loop ligation of parenchymal blebs and bullae. J Thorac Cardiovasc Surg 1997; 113: 50-54

$78 \mathrm{Kim} \mathrm{KH}$, Kim HK, Jan JY et al. Transaxillary minithoracotomy versus video assisted thoracic surgery for spontaneous pneumothorax. Ann Thorax Surg 1996; 61: 1510-1512

${ }^{79}$ Naunheim KS, Mack MJ, Hazelrigg SR et al. Safety and efficacy of video-assisted thoracic surgical techniques for the treatment of spontaneous pneumothorax. J Thorac Cardiovasc Surg 1995; 109: 1198-1204

${ }^{80}$ Khalife J, Avtan L, Feito B et al. Traitement des pneumothorax spontanés en vidéo-chirurgie: 32 observations. Chirurgie 1992; 118: $648-651$

${ }^{81}$ Suter M, Berner M, Vandoni R et al. Traitement par thoracoscopie du pneumothorax récidivant. Helv Chir Acta 1994; 60: 465-470

${ }^{82}$ Mouroux J, Benchimol D, Bernard JL et al. Traitement chirurgical du pneumothorax par vidéo-thoracoscopie. Presse Med 1992; 21: $1079-1082$

${ }^{83}$ Freixinet J, Canalis E, Rivas JJ et al. Surgical treatment of primary spontaneous pneumothorax with video-assisted thoracic surgery. Eur Respir J 1997; 10: 409-411

84 Tanaka F, Itoh M, Esaki $\mathrm{H}$ et al. Secondary spontaneous pneumothorax. Ann Thorac Surg 1993; 55: 372-376

${ }^{85}$ Harrison Jr. LH. In some cases - avoid talc pleurodesis. Chest 1995; 108: 289

${ }^{86}$ Judson MA, Sahn SA. The pleural space and organ transplantation. Am J Respir Crit Care Med 1996; 153: 1153-1165

87 Schramel FMNH, Sutedja TG, Braber JCE et al. Cost-effectiveness of video-assisted thoracoscopic surgery versus conservative treatment for first time or recurrent spontaneous pneumothorax. Eur Respir J 1996; 9: $1821-1825$

88 Schramel FMNH. Treatment of spontaneous pneumothorax - an ongoing debate. Eur Respir J 1998; 11: 514 - 515 (reply)

89 LeChevallier B, Jehan A, Gallet E et al. Le talcage pleural par thoracoscopie dans le pneumothorax du grand insuffisant respiratoire obstructif. Rev Mal Resp 1987; 3: 215-217

${ }^{90}$ Lee P, Wee SY, Wee YP et al. An audit of medical thoracoscopy and talc poudrage for pneumothorax prevention in advanced COPD. Chest 2004; 125: $1315-1320$

${ }^{91}$ Light RW. Pneumothorax. In: Pleural diseases, 5th edition. Baltimore: Williams \&Wilkins, 2002: 242-277

${ }^{92}$ Frank W, Loddenkemper R. Pleural disease: Pneumothorax and bronchopleural fistula. London: Arnold, 2002: 229-234

\section{Bereits publizierte Beiträge zu dieser Serie:}

${ }^{1}$ Bildgebende Diagnostik bei Pleuraerkrankungen. Pneumologie 2004; 58: $238-254$

2 Pleura: Pathologie nicht-neoplastischer Erkrankungen. Pneumologie 2004; 58: 516-524

${ }^{3}$ Pleuramesotheliom - Pathologie und Pathogenese. Pneumologie 2004; 58: 670-679

${ }^{4}$ Diagnostisches Vorgehen beim Pleuraerguss. Pneumologie 2004; 58 : $777-790$

${ }^{5}$ Management der malignen Pleuraergüsse. Pneumologie 2005; 59: $120-135$

${ }^{6}$ Therapeutische Optionen beim malignen Pleuramesotheliom. Pneumologie 2005; 59: 337-348

${ }^{7}$ Die Behandlung des parapneumonischen Ergusses und des Pleuraempyems. Pneumologie 2005; 59: 696-703 